\title{
VII.
}

\section{Beitrag zur Lehre von der Legis actio sacramento in rem.}

\author{
Von \\ Herrn Amtsrichter E. Roth \\ in Lauenburg a. d. E.
}

Der Zweck dieser kleinen Abhandlung ist eine von der bisher herrschenden Ansicht abweichende Meinung über die Vindikation aufzustellen, jedoch schicke ich als Einleitung eine kurze Darstellung der Entstehung der Legis actio sacramento in rem voraus, wie ich dieselbe mir vorstelle.

Die Legis actio sacramento ist meiner Ansicht nach aus einer Anrufung eines priesterlichen Schiedsgerichts der Pontifices entstanden und zwar glaube ich, dass die Zeit dieser Entstehung weit in die Zeit der Könige hineinreicht. Beide Parteien erschienen vor dem Forum der Priester, Beklagter stellte eidlich das Recht des Klägers in Abrede, Kläger beschwor es, worauf die Pontifices den Fall untersuchten und entschieden, cujus sacramentum justum, cujus injustum esset. Hiermit wurde indirekt auch über das beschworene Recht erkannt. Da nun der falsche Eid ein Frevel gegen die Götter war, so musste derselbe gesühnt werden und deshalb stellte jede Partei, je nach dem Objekt, um welches es sich handelte, eine Anzahl Opferthiere in das Heiligthum, welche im Fall des Unterliegens den Göttern geschlachtet wurden. Später trat an die Stelle der Opferthiere eine Geldsumme, welche dem Heiligthume verfiel. In beiden Fällen zog der Sieger das von ihm Hinterlegte zurück.

Ihering (Geist des r. R. Bd. I S. 300-307), welcher übrigens zweifelhaft geworden ist, hat diese Ansicht, dass sacramentum ursprünglich einen Eid bedeutet habe, zuerst aufgestellt. Danz (der sakrale Schutz im r. Rechtsverkehr S. 151-190), Karlowa (Römischer Civilprocess S, 14-29) 
und Huschke (Multa und Sacramentum Cap. VI) haben sie ausführlich vertheidigt, die meisten übrigen Rechtshistoriker stehen mit Bekker (Aktionen Bd. I S. 61 Note) auf dem Standpunkt des Non liquet. Dass grade die Pontifices in Verbindung mit dieser Entstehung der Legis actio sacramento $\mathrm{zu}$ denken sind, glaube ich daher, weil sie nach der römischen Geschichte in einem sehr engen Verhältniss zu der Rechtsprechung der alten Zeit gestanden haben und weil sie es waren, in deren Schule die römischen Juristen erzogen wurden. (Cf. Ihering: Geist d. r. R. Bd. II S. 381; Bekker: Aktionen Bd. I S. 68; Cic. Orat. I, 43; Varro: L. L. V. 180. Ea pecunia, quae in judicium venit in litibus, sacramentum a sacro. Qui petebat et qui infitiabatur.... quingenos aeris ad pontem deponebant $u$. fg.). Aus rein theoretischen Studien können sie diese Rechtskenntniss nicht gewonnen haben, und durch solche allein konnte ihr Einfluss nicht entstanden sein, das widerspricht der ganzen Rechtsentwickelung der Römer, welche auf der Praxis beruht. Nimmt man aber an, dass sie mit der Entstehung der Legisactionen in Verbindung gestanden haben, so erklärt es sich, wenn sie, auch nachdem sie aus der Praxis verdrängt waren, durch die auf älterer Praxis beruhende, in ihrem Kollegium weitergepflegte Theorie einen gewaltigen Einfluss auf die Rechtspflege ausübten; es erklärt sich ferner, was sonst kaum erklärlich ist, wie sie in Besitz der Legisactionsformeln kamen. Die Praxis konnten sie aber wohl nicht anders haben, als wenn sie in ältester Zeit als Schiedsrichter von beiden Parteien angerufen fungirten,. denn die eigentlichen Richter waren in älterer Zeit die Könige, später die Konsuln. Bei deren vielfacher Verhinderung und dem Ansehen der Pontifices mochte es aber häufig vorkommen, dass die Parteien sich deren Spruch freiwillig unterwarfen, und als Mittel hierzu diente der Eid, das heisst die feierliche Anrufung der Götter unter Behauptung eines Rechts. Widersprachen zwei solche Anrufungen der Götter einander, so war offenbar ein Frevel geschehen und die Priester mussten untersuchen, wer den Frevel begangen hatte und sich entsühnen müsse, wodurch gleichzeitig über das eidlich behauptete Recht entschieden wurde. Aus Vorstehendem ergiebt 
sich, dass die Behauptungen beider Parteien sich direkt widersprechen mussten, denn nur in diesem Falle lag klar ein Frevel vor, durch dessen Vorhandensein eine Entscheidung der Priester nothwendig wurde. Deshalb meine ich, dass nicht jede Partei ihr Recht unter Anrufung der Götter betheuerte, sondern dass nur Kläger sein Recht beschwor, während Beklagter es eidlich in Abrede stellte, denn nur in diesem Falle war ein Resultat, nach dem beide Theile Unrecht hatten, unmöglich; und ausserdem spricht auch der Umstand dafür, dass später der Sakramentsprocess den Charakter einer Wette angenommen hat, in welcher sich die Behauptungen der Parteien direkt widersprechen müssen. Dafür spricht auch der Wortlaut der Formeln, denn die Aufforderung zur Wette lautet bei der Legis actio sacramento in rem: Quando tu injuria vindicavisti, also wenn du Unrecht hast, und bei der Legis actio sacramento in personam lautet die Formel: Quando negas .... Quando ais egoque abs te captus sum.

An die Stelle der feierlichen Anrufung der Götter traten später einfachere Formeln. Beklagter bestritt das Recht des Klägers, dieser behauptete es und beide Parteien deponirten die durch feste priesterliche Satzung für jeden Fall bestimmte Sakramentssumme, welche im Falle des Unterliegens dem Heiligthume verfiel. Auf diese Weise hatte der Process den Charakter einer Wette angenommen und unter sacramentum verstand man nicht mehr die feierliche Anrufung der Götter, sondern das für den Fall des Unterliegens den Göttern geweihte Sühngeld. Weil dieser von den priesterlichen Schiedsrichtern ausgebildete Process sich praktisch bewährt hatte, nahm ihn der Staat auf und auf dieser Aufnahme eines von ihnen geschaffenen Instituts sowie ihrer langjährigen Thätigkeit als Schiedsrichter beruhte meiner Ansicht nach der Einfluss der Pontifices auf die Rechtsprechung der älteren Zeit. Das Wort sacramentum in dem verstaatlichten Sakramentsprocess verlor nun natürlich gänzlich seine Beziehung auf den Fid und erhielt die Bedeutung einer für den Fall des Unterliegens dem Staat zufallenden Wettsumme (cfr. Puchta: Inst. Bd. II S. 87, welcher die sponsionis summa das verweltlichte sacramentum nennt). Dieses wurde auch häufig 
nicht mehr baar hinterlegt, sondern es wurden Bürgen dafür gestellt (Gai. IV, 16).

Die Haupteigenthümlichkeit der Legis actiones, wie sich dieselben nach der Abfassung der XII Tafeln gestalteten, war, wie Ihering (Geist d. r. R. Bd. II S. 612) ausführt, dass die Klageformeln wörtlich dem Gesetz nachgebildet wurden (actiones .... . legis actiones appellabantur ideo, quia ipsarum legum verbis accommodatae erant et ideo immutabiles proinde atque leges observabantur. Gai. IV, 11). Es gab so viel Legis actiones als einzelne selbstständige Artikel des Gesetzes, welche einen Rechtsanspruch gewährten. Wir haben heut zu Tage eine Analogie in den meist wörtlich dem Gesetz entsprechenden Anklageformeln und thatsächlichen Feststellungen im Strafprocess. Die Legis actiones enthielten wie sie eine in die Worte des Gesetzes gefasste kurze Darstellung des Sachverhältnisses. An welcher Stelle im Verfahren wurde aber diese aus dem Gesetz geschöpfte Formel gesprochen? Ich glaube, dass uns die Formel der vindicatio darauf führt. Der erste Vindikant sprach: Postulo anne dicas, qua ex causa vindicaveris, er fragte also den Gegner nach dem gesetzlichen Grunde seines Anspruchs. Dieser antwortete: Ius peregi sicut vindictam imposui. Hier berief er sich also auf die vorauf gegangene Vindikation. Nimmt man an, dass diese Worte, welche doch offenbar einen wesentlichen Bestandtheil der Formel gebildet haben, die der Wette vorausgehende Fixirung des Streitpunktes enthalten sollten, so wird bei den Legis actiones, mit welchen keine Vindikation verbunden war, an dieser Stelle Kläger, vom Beklagten gefragt, sich mit den Worten des Gesetzes auf sein Recht berufen haben. Dass dies der Platz der eigentlichen Legis actio ist, dafür spricht auch die Formel der 1. a. s. in personam, bei welcher freilich die entscheidenden Worte fehlen: Quando negas, sacramento quingenario te provoco. Adversarius quoque dicebat: Quando ais egoque abs te captus sum. Es muss hiernach den Worten: Quando negas ein Bestreiten, ein nego, vorausgegangen sein, und diesem wiederum ein Behaupten des streitigen Rechts, ein ajo. Bei der l. a. s. in rem genügte an dieser Stelle eine Bezugnahme auf die vorangegangene Vindikation, da diese eben, wie ich weiter 
unten ausführen werde, nichts weiter war als eine feierliche Eigenthumsbehauptung, und die Frage: Postulo anne dicas war nur nothwendig, um eine Grundlage für die Wette $\mathrm{zu}$ gewinnen.

Bei der Legis actio sacramento in rem tritt dem Sakramentsverfahren die Vindikation hinzu. Die Form des ganzen Verfahrens hat uns Gajus (IV, 16) überliefert. Der Vindikant berührte mit einem Stabe, welcher die hasta, das Symbol des civilrechtlichen Eigenthums, darstellen sollte, den Gegenstand der Vindikation, welcher zu dem Zwecke ganz oder durch einen Theil repräsentirt vor Gericht geschafft wurde, und sprach die Worte der Vindikation. Der Gegner kontravindicirte. Der Prätor stiftete Frieden. Vindikant fragte nach dem Rechtsgrunde der Kontravindikation, welcher ihm mitgetheilt wurde, worauf er die Wette anbot. Zum Schluss ertheilte der Prätor einer Partei die Vindicien d. h. den vorläufigen Besitz gegen Stellung von Bürgen. Soweit sind Alle über den Vorgang einig, im Uebrigen aber herrscht viel Streit.

Manche meinen, die Parteirollen seien von Anfang an fixirt, und zwar Vindikant sei Kläger, Kontravindikant Beklagter gewesen (Puchta, Institutionen $\S 161 \mathrm{~m}$. S. 472, 9 A.). Allein es ist dann nicht begreiflich, weshalb Beklagter sich nicht mit einer Negation begnügte, sondern kontravindicirte.

Andere (Wetzell: Vindikationsprocess S. 7, 60; v. Bethmann-Hollweg, Röm. Civilpr. Bd. I \& 40 und 42, namentlich S. 144; Keller, Civilpr. S. A 210 S. 64 (Wach); Huschke, Gajus S. 188 und Multa und Sacr. S. 459; Bekker, Aktionen Bd. I S. 209) stellen die Behauptung auf, durch die Vindicienertheilung seien die Parteirollen fixirt, das Verfahren sei aus einem zweiseitigen ein einseitiges geworden, der Nichtbesitzer habe alsdann sein Eigenthum beweisen müssen. Dagegen spricht, dass nicht recht einzusehen ist, weshalb dann der Prätor die Vindicien nicht unmittelbar nach der Vindikation ertheilte und das weitere einseitige Verfahren danach richtete, wer die Vindicien erhalten hatte und wer nicht, während nach Gajus auch im zweiten Theil der Formel zwischen dem, qui prior vindicaverat und seinem Gegner unterschieden wird und die Vindicien erst nach Schluss des Verfahrens ertheilt werden. 
Ihering (Geist d. r. R. Bd. III S. 90-106); Eck, (Doppels. Klagen S. 7-19) und Karlowa (Röm. Civilpr. S. 69-88) nehmen eine vollkommene Duplicität an. Beide Theile mussten nach ihnen gleichmässig beweisen, standen sich gleichberechtigt gegenüber, nicht wie Kläger und Beklagter, sondern wie Kläger und Widerkläger, die contravindicatio ist nach ihnen eine reconventio necessaria. Am Schluss entschied nach ihrer Ansicht der Richter über beide sacramenta und damit über die Behauptungen beider Parteien. Hierbei entsteht nun die Frage, was geschah, wenn keine von beiden Parteien ihre Behauptungen erwies? Eck (S. 16) meint, der Richter habe alsdann zu Gunsten desjenigen entschieden, welcher das relativ bessere Recht, beziehungsweise den jüngsten Besitz dargethan habe; die Römer hätten in jener Zeit den Eigenthumsbegriff noch nicht in der späteren korrekten Weise erfasst, die Eigenthumsklage habe gleichsam die Publiciana mit umfasst. Allein dagegen spricht der klare Wortlaut der Formeln: Beide behaupten: Rem meam esse ex jure Quiritium. Es spricht auch der ganze Entwickelungsgang des Rechts dagegen, nach dem in ältester Zeit der Eigenthumsbegriff viel schärfer entwickelt war als später. lhering (S. 92) und Karlowa (S. 86) nehmen deshalb an, dass der Richter, wenn Keiner vollen Beweis führte, beide sacramenta für injusta erklärt habe, und dass, wer den Besitz hatte, ihn behielt. Allein wir erhalten dann zwei Besiegte und keinen Sieger, und dagegen spricht Gai. IV, 13: Qui victus erat, summam sacramenti praestabat poenae nomine eaque in publicum cedebat und Varro L. L. V. 180: Qui judicio vicerat, suum sacramentum e sacro auferebat, victe victi ad aerarium redibat. Ueberdies spricht die später zu besprechende zweite Hälfte der Legisaktionsformel durchaus gegen die Duplicität der Legis actio sacramento in rem.

Eine ganz neue Theorie hat Lotmar (Zur Legis actio sacramento in rem, namentlich S. 18 und: Kritische Studien in Sachen der Kontravindikation) aufgestellt. Während bisher Alle die contravindicatio als Eigenthumsgegenbehauptung ansahen, fasst er diese als nur mögliche, nicht nothwendige Form der contravindicatio auf, lässt also eine feierliche Verneinung ohne Behauptung des Eigenthums seitens des Be- 
klagten als möglich zu. Damit kommt er dahin, dass er als den Kern der vindicatio das Auflegen der festuca, ,das Gewalt zeigen": annimmt. Mit Recht wendet Brinz (Zur Kontravindikation der Legis actio sacramento S. 103) dagegen ein, dass schon im Gebrauche der festuca als Eigenthumssymbol an sich eine Berufung auf das Recht, eine Eigenthumsbehauptung liege, wie Gajus sagt (IV, 16): Festuca autem utebantur quasi hastae loco signo quodam iusti dominii, quod maxime sua esse credebant, quae ex hostibus cepissent. Lotmars Theorie widerspricht ganz entschieden den Quellen, namentlich dem Gajus, welcher ausdrücklich sagt: Adversarius eadem similiter dicebat et faciebat. Kontravindikant sprach also: Hunc ego hominem ex jure Quiritium meum esse ajo, d. h. er behauptete Eigenthum. Hätte er Eigenthum des Vindikanten bestritten, so hätte er nicht dasseibe gesprochen wie dieser, sondern das Gegentheil. Das Zeugniss des Gajus wird Lotmar trotz allen von ihm aufgewendeten Scharfsinns nicht wegschaffen und ein Missverständniss des Gajus, welcher sich durchaus klar in diesem Falle ausspricht, annehmen, das darf man doch nur, wenn es keine Möglichkeit giebt, es zu erklären, weshalb bei den Römern eine beiderseitige Eigenthumsbehauptung nothwendig war.

In Folge des Suchens nach einer Erklärung dafür, dass beide Parteien Eigenthum behaupteten, ist Brinz in der schon angeführten Abhandlung unter Bezugnahme auf Du Roi (Arch. f. civil. Pr. Bd. VI S. 264), Zimmern (Gesch. d. r. Privatr. Bd. III S. 108) und Wetzell (Vindikationsprocess S. 45) dahin gelangt, dass er die Theorie aufstellt: Beide Parteien hätten den Vindikationsprocess gleichmässig um Besitz und Eigenthum gekämpft, der Besitz sei vor der Vindikation als gänzlich unentschieden zu betrachten, die Sache habe frei im Gericht gelegen, beide Parteien hätten sie gleichmässig beansprucht und hätten deshalb auch beide vindiciren müssen. Brinz kommt also $\mathrm{zu}$ einer actio petitoria gegen einen Nichtbesitzer. Schwierigkeiten macht ihm (S. 137) der Fall, wenn der Besitz unstreitig war, es also eines gleichzeitigen Streites um Besitz und Eigenthum nicht bedurfte. Brinz stellt die Vermuthung auf, dass in diesem Fall eine 
Legis actio per judicis arbitrive postulationem Platz gegriffen habe, allein der Fall, dass der Besitz unstreitig war, war sicher nicht der Ausnahmefall, sondern die Regel, und wenn eine Theorie für diese nicht passt, so ist sie nicht richtig. In den Quellen ist auch nichts von einer Legis actio per judicis arbitrive postulationem erwähnt, während dies sicher der Fall wäre, wenn sie in dem häufigen Fall unstreitigen Besitzes angewendet wäre, wie Gai. IV, 91 ausdrücklich die beiden nebeneinander bestehenden Formen der actio in rem aufgeführt sind. Wenn bei der l. a. s. in rem Keiner als Besitzer angesehen wurde, so mussten doch auch beide Parteien im Verlauf des Processes gleichmässig behandelt werden, was Wetzell wenigstens nicht annimmt und was auch der zweiten Hälfte der Formel widerstreitet.

Durch die Abhandlungen von Lotmar und Brinz kann soviel meiner Ansicht nach als festgestellt gelten, dass die bisherigen Erklärungen der Kontravindikation ungenïgend sind, und deshalb wage ich es zu unternehmen, eine neue Ansicht aufzustellen.

Unter vindicere, vindicare versteht Lotmar (Zur Legis actio S. 25) lediglich ein "Gewalt zeigen"; ein Eigenthumsbehaupten, meint er, habe in alter Zeit nicht darin gelegen. Das vindicere, das Gewalt zeigen, muss doch aber einen Sinn gehabt haben und die Antwort, welcher dies sei, bleibt Lotmar schuldig. Die von O. Müller (Rh. Museum Bd. V S. 190-197) aufgestellte etymologische Ableitung mag richtig sein, immer aber enthält das vindicere nicht nur ein Gewalt zeigen durch die That, sondern auch ein Gewalt zeigen mit Worten (Ihering: Geist d. r. R. Bd. I S. 163 Anm. 69; v. Bethmann-Hollweg: Röm. Civilpr. Bd. I $\S 40$; Wetzell : Vind. S. 47; Gellius IV, 17 : Quod videtur dixisse, conferens vim illam civilem et festucariam, quae verbo diceretur, non quae manu fieret, cum vi bellica et cruenta), ein Gewalt Behaupten, und es scheint mir diese Bedeutung sogar die Hauptbedeutung zu sein, denn vim dicere heisst ja übersetzt einfach Gewalt behaupten, wenn auch dicere auf denselben Grundstamm, wie $\delta \varepsilon \iota x \nu v v \alpha \iota$, zurückzuführen ist. Die mit dem Gewaltbehaupten verbundene symbolische Gewalt scheint mir die Nebensache 
zu sein, die Form, welche erst durch die hinzugefügten Worte Bedeutung gewinnt und deren Sinn eben durch die Worte ausgedruickt wird. Unter vindicere, vindicare verstehe ich also ein Berufen au die Gewalt mit solennen Worten und durch eine symbolische Handlung. Auf was für eine Gewalt konnte sich aber der Vindikant berufen? Er nahm die volle und ganze Gewalt über die vindicirte Sache in Anspruch, er behauptete, dass er allein Gewalt über die Sache auszuüben und jedes Dritten Einwirkung auszuschliessen berechtigt sei; deshalb legte er die Lanze, zugleich als Symbol der Abwehr und des Angriffs, auf den Gegenstand der Vindikation. Das Recht der vollen Gewalt über die Sache ist aber das Eigenthumsrecht. Der Vindikant berief sich also auf die Gewalt, welche der Eigenthümer über den Gegenstand des Eigenthums jedem Dritten gegenüber hat. Das ist der Sinn der Worte und der Handlung. Hunc ego hominem ex jure Quiritium meum esse ajo lautet die Formel bei Gajus, ihr Inhalt ist also nichts als eine feierliche Eigenthumsbehauptung.

Die actiones in rem haben zwar ibre Richtung zunächst auf die Sache, aber nicht auf die herrenlos im Gericht liegende Sache, sondern auf die von Jemand vertretene Sache. Erst dadurch, dass Jemand das Recht an der Sache, welches der Kläger beansprucht, stört, wird die actio in rem begründet. Solange keine Störung vorliegt, ist zwar ein Recht vorhanden, aber keine Klage. Der Beklagte kann zwar die Folge in jus nicht weigern, aber er braucht sich auf die Klage nicht einzulassen, es giebt keinen Zwang für ihn zur Uebernahme des Processes bei den actiones in rem. (Bekker: Aktionen Bd. I S. 202 L. 80 D. 6, 1: In rem actionem pati non compellimur). Durch Anerkennung des Rechts entgeht er der Verfolgung, soweit nicht etwa ein mit der Klage verbundener persönlicher Anspruch in Frage kommt. Dieser allgemeinen Regel unterliegt auch die Vindikation. Sie dient speciell zum Schutz der positiven Seite des Eigenthums, soll dem Eigenthümer die Möglichkeit schaffen, selbst beliebig auf die Sache einzuwirken, und richtet sich deshalb gegen Denjenigen, welcher die Einwirkung des Eigenthümers auf die Sache ausschliesst 
E. Roth,

(L. 25 p. D. 44, 7; Gai. IV, 51). Diese Richtung der Klage gegen den Besitzer gründet sich auf den Zweck der Klage, die positive Seite des Eigenthums zu schützen, sie wird deshalb zur Zeit der Legisaktionen so gut gegolten haben wie später (cfr. Lotmar: Zur Legis actio S. 77). Die Legis actio sacramento in rem war sogar noch mehr beschränkt als die spätere Vindikation, wie dies die aus den Quellen von Lotmar noch nicht weginterpretirte Contravindicatio ergiebt, indem sie nur gegen den ging, welcher seinerseits Eigenthum beanspruchte. Der Grund hierfür wird wohl darin gelegen haben, dass der Prätor nach dem wirklich streitigen Recht die anzustellende Klage bestimmte, also in dem Klagezwang, wie ihn Ihering (Geist d. r. R. Bd. III Bl. 83 fg.) annimmt. Jedenfalls erlaubt der Umstand, dass der Beklagte, mag dies nun der Vindikant oder Kontravindikant sein, behauptete: Hunc ego hominem ex jure Quiritium meum esse ajo, den logischen Schluss, dass die Sakramentsklage nur gegen den ging, welcher selbst Eigenthum beanspruchte.

Der in jus vocatus war, wie oben erwähnt, nicht genöthigt, sich auf die Klage einzulassen. Damit also die L. a. s. in rem angestellt werden konnte, war zunächst erforderlich, dass ein Beklagter vorhanden war, welcher die Einwirkung des Klägers auf die Sache ausschloss und Eigenthum an der Sache beanspruchte, auch den Willen dokumentirte, dies beanspruchte Recht vor Gericht zu vertheidigen. Die dingliche Klage ging nach der Anschaung der Römer gegen die Sache selbst und nur dadurch gegen den Beklagten, dass dieser in eine bestimmte Beziehung zur Sache trat. That der in jus vocatus dies nicht, liess er ruhig zu, dass Kläger sich der Sache bemächtigte, so war er nicht der rechte Beklagte und eine Sakramentsklage war unmöglich. Nichts war also natürlicher, als dass das Verfahren damit begann, dass Beklagter in äussere Beziehung zur Sache trat und durch Wort und Werk die Absicht dokumentirte, sich auf die Klage einzulassen, kurzum, dass er sich, wie beim Zweikampf, dem Gegner stellte und sich als richtiger Beklagter durch Behauptung des Eigenthums einführte. Dies geschah meiner 
Ansicht nach durch die Vindikation. Vindikant, welchen ich also für den Beklagten, nicht für den Kläger ansehe, sprach die Worte der Vindikation, e r betheuerte feierlich sein Eigenthum und legte zum Zeichen, dass er die volle Gewalt über die in seinem Besitz in forobefindliche $S$ ache beanspruchte, die festuca, früher die hasta auf. Er ist offenbar von den Beiden der Besitzer, sonst würde er nicht zuerst die hasta auflegen, und dies spricht auch schon dafür, dass er Beklagter ist. Die hasta war in seinen Händen ein Symbol der Abwehr, ein Zeichen, dass er den Gegner vom Besitz ausschloss. Die an den Kläger gerichteten Worte Ecce tibi vindictam imposui bedeuteten für ihn, ich habe Dir, um Dich abzuhalten, die vindicta aufgelegt. Damit hatte sich Beklagter feierlich als rechter Beklagter im Sakramentsprocess gestellt. Nunmehr, nachdem ein Vertheidiger der Sache gefunden war, konnte Kläger seine Klage anstellen, er behauptete ebenfalls Eigenthum und berührte mit der festuca die Sache, wodurch der Streit formirt war. In Händen des Klägers war die hasta ein Symbol des Angriff's, und die Worte Ecce tibi vindictam imposui bedeuteten für ihn, ich habe Dir, um Dich anzugreifen, die vindicta angelegt. Nach der Vindikation folgte der Friedensbefehl des Prätors, die Frage des Beklagten nach dem Grunde der Klage, die Angabe desselben seitens des Klägers, die Aufforderung zur Wette über das Recht des Klägers seitens des Beklagten und die Annahme derselben. Zum Schluss des Verfahrens ertheilte der Prätor die Vindicien an eine der Parteien, $d$. h. er verlieh einer Partei während der Dauer des Processes den Besitz und schützte ihn durch Polizeimandat. Wurde das Mandat vom Gegner gebrochen, so kam Derjenige, welchem es der Prätor ertheilt hatte, zu diesem und erlangte von ihm nach stattgehabter Untersuchung über den Bruch einen Strafbefehl auf Schadensersatz und Wiederherstellung des Besitzes, falls er entzogen war. (Keller: Civilpr. § 22.) Der Umstand, dass die Vindicien erst nach Schluss des Verfahrens ertheilt wurden, scheint mir die Annahme unmöglich zu machen, dass sie auf das Verfahren selbst irgend welchen Einfluss gehabt haben, abgesehen davon, dass Grajus, wenn die Beweispflicht von der Ertheilung 
der Vindicien abgehangen hätte, dies unzweifelhaft erwähnt haben würde.

Wer aber erhielt die Vindicien? Erhielt sie der juristische Besitzer oder war der Prätor bei Ertheilung derselben nicht beschränkt? ${ }^{1}$ ) Meiner Ansicht nach hing die Entscheidung in ältester Zeit wesentlich vom Belieben des Prätors $a b$, wenn auch einige gesetzliche Regeln, z. B. dass die Vindicien im Freiheitsprocess zu Gunsten der Freiheit gegeben werden mussten, schon bestanden. Allmählich entwickelten sich indess feste Regeln, indem jeder Prätor die Voraussetzungen, unter denen er während der Dauer seines Amts die Vindicien und die Polizeimandate ertheilen wollte, im Edikt öffentlich bekannt machte, welche Voraussetzungen alsdann von einem Edikt in das andere übergingen. Aus der so von bestimmten Voraussetzungen abhängig gemachten Vindicienertheilung entstanden, wie ich mit Ihering und Bekker annehme, die Interdikte, indem der Prätor, wenn die Frage, wer Besitzer sei, verwickelter war, sie von dem Eigenthumsprocess trennte und vorher durch einen judex untersuchen und entscheiden liess. ${ }^{2}$ ) Ein Hauptgrund, der ihn zu solcher Trennung veranlassen konnte, war, dass es öfter vorkommen mochte, dass Kläger die Vindicien erhalten hatte,

1) Vergl. Thering: Beiträge zur Lehre vom Besitz in den Jahrb. f. Dogm. Bd. IX S. 58-70; Bruns: R. d. Bes. S. $38-53$ und Besitzkl. S. 17-26; Bekker: R. d. Bes. namentlich S. 92-104 und Aktionen Bd. I S. 209; Lotmar: Zur Legis actio S. 80 fg.; Brinz: Zur Contravindikation S. 130; Keller: Civilpr. $\$ 74 \mathrm{fg}$. Ein genaueres Eingehen in die Frage von der Entstehung der Interdikte würde über den Zweck dieser Abhandlung hinausführen, und ist dieselbe deshalb nur berührt. 2) Die Interdikte wurden, wenn ihre Voraussetzungen vorlagen, auch ertheilt, ohne dass eine Eigenthumsklage angestellt war. Namentlich trat dies wohl ein, wenn ein civiles Eigenthum überhaupt nicht vorhanden war, z. B. bei dem Besitz am ager publicus. Ob dies schon zur Zeit der Vindicien, als die Ertheilung der Polizeimandate lediglich auf Grund prätorischer Kognition erfolgte, geschehen ist oder erst spätex nach richterlicher Untersuchung wie zur Zeit der Interdikte, kann hier dahingestellt bleiben. Jedenfalls erklärt diese doppelte Verwendung der Interdikte zur Vorbereitung des Eigenthumsprocesses und als selbstständiges Rechtsmittel den noch immer nicht beendigten Streit über deren Entstehung und die juristische Natur des ihnen zu Grunde liegenden Besitzes. 
aber Eigenthum nicht nachweisen konnte, wohl aber redlichen Erwerb im Sinne des späteren Rechts - wenn auch nicht daran zu denken ist, dass die bonae fidei possessio zur Zeit des Sakramentsprocesses bereits als Rechtsinstitut bestanden hat - und juristischen Besitz, wie er Voraussetzung der Vindicien war, während Beklagter, wie sich aus der Beweisaufnahme ergeben mochte, keinerlei Recht hatte, obschon er die Unverfrorenheit besessen hatte, Eigenthum $\mathrm{zu}$ behaupten (Ihering: Beiträge S. 64). Das klägerische sacramentum musste alsdann für injustum, das des Beklagten für justum erklärt werden, und wenn der Prätor nicht einen anderen Weg fand, den Kläger trotz des misslungenen Eigenthumsbeweises als besser Berechtigten im Besitz zu schützen, etwa dadurch, dass er die praedes litis et vindiciarum nicht mit dem sacramentum für verfallen erklärte, so trug der minder Berechtigte als Beklagter und ursprünglicher, wenn auch nicht juristischer Besitzer den Sieg davon und musste in Besitz gesetzt werden. Liess aber der Prätor bei Streitigkeit des $z u$ den Interdikten berechtigenden Besitzes diesen Streit, bevor er die Eigenthumsklage zuliess, entscheiden, so konnte ein solcher Ausfall des Processes nicht so leicht vorkommen. Derjenige, welcher in dem Vorprocess gesiegt hatte, war dann als Beklagter und präsumptiv besser Berechtigter auch in der günstigeren Lage, den Ausfall des gegnerischen Beweises abwarten zu können. Nach Entscheidung der Besitzfrage und Ertheilung der Interdikte konnte freilich die Sakramentsklage mit der Vindikation nicht mehr angestellt werden, denn die Interdikte wären durch die vindicatio gebrochen worden, und wenn dies auch nur eine Form war, so würde ein solcher Bruch doch in zu krassem Widerspruch mit den Anschauungen der Römer, bei denen Form und Sinn sich decken mussten, gestanden haben. Es musste also nunmehr anders geklagt werden. Deshalb bin ich der Meinung, dass diese Verwendung der Interdikte zur Vorbereitung der Eigenthumsklage in die Zeit des Formularprocesses fällt und vielleicht der Grund für die Einführung der formula petitoria war. Fiel hiermit auch allmählich die alte Form der vindicatio fort, so blieb doch ihr Sinn, und weil Kläger auch bei der postulatio formulae den Prätor unter Berufung auf sein Eigenthumsrecht anrief, 
wurde für die Eigenthumsklage der technische Ausdruck Vindikation beibehalten.

Wie stimmen nun die verschiedenen Ansichten mit den Quellen überein? Auffällig ist zunächst und widerspricht der Lehre von der Duplicität direkt, dass im Gajus (IV, 16) keineswegs Vindikant und Kontravindikant gleich behandelt werden, wie dies Lotmar ausführlich nachweist. Vielmehr werden ausdrücklich die Worte: Postulo, anne dicas, qua ex causa vindicaveris, dem, qui prior vindicaverat, in den Mund gelegt, die Antwort spricht der Gegner, die Auftorderung zur Wette wird ausdrücklich dem Vindikanten zugetheilt. Ebenso wie Gajus hervorhebt, dass die Vindicien secundum alterum ertheilt seien, würde er auch irgend wie hervorgehoben haben, dass es gleichgültig sei, wer zuerst vindicirte und wer jedesmal die vorgeschriebenen Worte sprach. Es würde bei einer gleichen Behandlung der Parteien nach der Frage über den Grund der Vindikation des Kontravindikanten und seiner Antwort, diesem dieselbe Frage an den Vindikanten in den Mund gelegt sein müssen. Vindikant sagte ausdrücklich: Quando tu injuria vindicavisti, D. aeris sacramento te provoco, also er proponirte nicht über sein Recht, sondern über das des Kontravindikanten die Wette. Das sacramentum des Vindikanten kann also nur dann injustum gewesen sein, wenn Kontravindikant sein Recht bewies. Wenn dieser auf die Wettofferte erwiderte: Similiter ego te, so sagte er damit durchaus nicht, dass er eine Wette proponire, dass Vindikant mit Unrecht vindicirt habe, denn dies wäre nicht die Annahme der angebotenen Wette, sondern das Anbieten einer neuen gewesen, und es müsste dann doch noch irgend etwas von der Annahme beider Wetten im Gajus stehen. Schon aus der ganzen Natur der Wette, bei der sich stets direkt entgegengesetzte Behauptungen gegenüber stehen müssen, folgt, dass Kontravindikant die Wette über sein Recht annahm, dass es sich also nicht um das Recht des Vindikanten, sondern stets nur um das des Kontravindikanten handelte. Lotmar (Zur Legis actio S. 55 fg., Kritische Studien S. 88) hat dies ausführlich dargethan und auch Brinz (S. 113) kann sich der Schwere der Argumente nicht verschliessen. Das Similiter ego te war An- 
nahme der Wette, auch wenn die dahinter stehenden unleserlichen Buchstaben etwa als ein undentliches Zeichen für etc. aufzufassen sind und wenn das Similiter ego te durch D. aeris sacramento provoco zu ergänzen ist. Bei einer Wette liegt immer ein gegenseitiges Provociren vor, Jeder fordert den Anderen heraus durch die Wettsumme, aber es dreht sich doch nur um eine Behauptung, und insofern enthält die Provokation des Herausgeforderten eine Annahme. Wenn Brinz meint, in der faktischen Ausführung, also in der Deponirung der Gelder von beiden Seiten, könne die Annahme erblickt werden, so ist zwar nicht zu bestreiten, dass eine Annahme auch in konkludenten Handlungen liegen kann, allein bei der formellen Natur der Sakramentsklage ist doch mit Sicherheit anzunehmen, dass, wenn zwei Herausforderungen und Annahmen stattgefunden hätten, die beiden Annahmen nicht durch konkludente Handlungen allein, sondern auch in Worten ihren Ausdruck gefunden hätten. Brinz (S. 114) weist dann auch selbst darauf hin, dass wenn zwei Wetten in Frage ständen, vier Sakramentssummen hätten deponirt werden müssen, und dass nur zwei deponirt seien, wie Gai. IV, 16 ergiebt: ab utroque praetor praedes accipiebat sacramenti, nicht sacramentorum. Er meint, jede Partei habe nur auf ihre Behauptung gesetzt und es sei nur ein Einsatz von Succumbenzgeldern, allein dem widerspricht die Aufforderung, denn Vindikant setzt die Sakramentssumme nicht ein für den Fall, dass er selbst Unrecht habe, sondern er provocirt den Kontravindikanten zum Einsetzen und durch Einsetzen der Sakramentssumme durch die Behauptung, dass jener Unrecht habe. Von Einsetzen von Succumbenzgeldern kann doch nur die Rede sein, wenn jede Partei den Betrag für den Fall ihres Unterliegens, ihres Unrechts einsetzt; die Wette aber charakterisirt sich grade dadurch, dass jede Partei behauptet, der Gegner habe Unrecht. Es kommt dies allerdings beides oft auf eins hinaus, im vorliegenden Fall aber nicht, denn wenn Vindikant wettet, dass der Kontravindikant Unrecht habe, so verliert er sein sacramentum nur, wenn dieser sein Eigenthum beweist; wenn er aber eine Strafsumme einsetzt für den Fall, dass er Unrecht habe, so muss er sein sacramentum verlieren, sobald er sein Eigen- 
thum nicht darthut, mag Kontravindikant Eigenthümer sein oder nicht.

Der innere Grund, weshalb die aus dem Gajus klar hervorgehende Einseitigkeit der zweiten Hälfte der Formel bestritten wird, ist auch nur der, dass dieselbe in scheinbar unlöslichem Widerspruch mit der Doppelseitigkeit der ersten Hälfte steht. Dieser Grund verführt die meisten Schriftsteller dazu, die Wegschaffung der Einseitigkeit der zweiten Hälfte durch allerhand Künste zu versuchen, und er verführt Lotmar umgekehrt dazu, die Nothwendigkeit der Eigenthumsbehauptung beider Parteien in der ersten Hälfte anzugreifen. Ich ziehe daraus, dass keine doppelte Wette erwähnt wird, sondern nur eine einzige, den Schluss, dass nicht $z$ wei Fragen, über das Recht des Klägers und des Beklagten, in Betracht kommen, sondern dass nur eine, über das Recht des Klägers, zu beantworten war. Da nun Vindikant zur Wette über das Recht oder Unrecht des Kontravindikanten auffordert, so muss man weiter logisch schliessen, dass Kontravindikant Kläger war, denn in erster Linie musste jedenfalls Kläger und nicht Beklagter sein Recht darthun. Daraus folgt aber weiter, dass Beklagter die erste Vindikation sprach. Dies erklärt sich auch völlig, wenn man annimmt, dass die Vindikation nichts weiter ist, als eine solenne Eigenthumsbehauptung beider Theile, wie sie weiter oben dargestellt ist. Die grösste Schwierigkeit bildet die Erklärung des Umstandes, dass nicht Kläger den Beklagten, wie bei den Legis actiones sacramento in personam, sondern Beklagter den Kläger zur Wette aufforderte. Allein auch dies lässt sich erklären. Bei den persönlichen Klagen hat der Beklagte keinerlei Interesse eine Entscheidung hervorzurufen, dagegen liegt, wenn Jemand Eigenthum an einer in fremdem Besitz befindlichen Sache beansprucht, dem Besitzer viel daran, die Bedrohung seines Rechts, welche dasselbe entwerthet, $\mathrm{zu}$ beseitigen. In ältester Zeit waren auch die Besitzinterdikte noch nicht selbstständig vorhanden, sondern ein integrirender Bestandtheil der Eigenthumsklage, also musste sich bei Besitzstörungen auch der im Besitz befindliche Eigenthümer durch in jus vocatio und vindicatio vor dem Prätor behufs Erlangung der Inter- 
dikte helfen, er war also, wenn der Gegner kontravindicirte, schon um die Interdikte zu erlangen, wesentlich dabei interessirt, dass die Sache zum Austrage kam. Für die Ansicht, dass nur das Recht des Klägers zur Entscheidung stand und dass Kläger der Kontravindikant war, spricht auch die Frage des Vindikanten: Postulo anne dicas, qua ex causa vindicaveris? und die Antwort darauf: Jus peregi, sicut vindictam imposui. Diese stehen meines Erachtens, wie ich bereits oben (S. 124) angeführt, an der Stelle der eigentlichen Legis actio und enthalten in Anknüpfung an die vorhergegangene Vindikation die Berufung des Klägers auf sein Recht, die Fixirung des Streitpunktes, welche der Wette vorausging. Die Berufung auf die Vindikation musste also seitens des Klägers erfolgen und in der That beruft sich der Kontravindikant und nur dieser allein auf dieselbe.

Die zweite Stelle, in welcher eine Vindikation erwähnt wird, ist Plautus Rud. 4, 3, 86. Trachalio: Hunc meum esse ajo. Gripus: Et ego item esse ajo meum. Lotmar (Zur Legis actio S. 14) macht bereits darauf aufmerksam, dass Trachalio im Besitz des streitigen Gegenstandes und deshalb Beklagter ist. Hier spricht also Beklagter die Vindikation und Kläger die Kontravindikation. Plautus macht allerdings auf juristische Genauigkeit keinen Anspruch, wie von Bethmann-Hollweg (Civilpr. Bd. I S. 34) in einer kurzen Kritik der römischen Schriftsteller und ihres Werths für die Rechtsforschung sagt, allein er fügt hinzu, er kleidet die Rechtsfälle in seinem Publikum vom Forum her wohlbekannte Formen. Damit hat er offenbar sagen wollen, dass er in den wesentlichsten, allen bekannten Hauptsachen nicht ohne Werth für die Rechtsforschung ist, aber im Detail sich allerlei dichterische Freiheiten erlaubt. Nimmt man dies Urtheil als richtig an, so muss man die hier in Frage stehende Stelle allerdings für bedeutsam halten, denn die Form der Vindikation als eines der wesentlichsten Rechtsmittel war in ihren Grundzügen gewiss allgemein bekannt, wie denn in Rom überhaupt zur Zeit des Plautus jeder gebildete Mann die Fundamente des Processes kannte. Danach scheint es mir allerdings für meine Ansicht zu sprechen, dass bei Plautus der Beklagte die Vindikation und Kläger die Kontravindikation spricht, zumal zu seiner 
Zeit, wie die gleich zu besprechende Stelle des viel späteren Cicero ergiebt, die Vindikation sicher noch bäufig in der alten Form vorkam.

Eine dritte Stelle findet sich bei Cicero: Pro Murena Cap. XII, 25 und 26. Lotmar (Zur Legis actio S. 15) meint, Cicero schlüge hier als Verfahren vor: Fundus Sabinus meus est; immo meus; deinde judicium, ohne dass dies Verfahren in Geltung gewesen wäre. Betrachtet man die Stelle unabhängig von den übrigen Quellenstellen, welche über die Vindikation sprechen, so ist solche Auslegung vielleicht möglich. Allein da wir in Gajus eine ausführliche Darstellung der Vindikation haben, in welcher eine beiderseitige Eigenthumsbehauptung vorkommt, müssen wir uns nach einer anderen Deutung umsehen und diese liegt sehr nahe. Cicero will in den wenigen Worten den Kern der Sache darstellen und zeigen, dass es nicht der von ihm gegeisselten Vielrederei bedurfte, um zum Ziele zu gelangen, sondern dass der derselben zu Grunde liegende Gedanke sehr einfach sei. Liest man die Stelle im Zusammenhang mit dem Vorhergehenden, so spricht Cicero folgende Gedanken aus: Bei dem älteren Verfahren hätten wenige Rechtskundige die Grundsätze des Rechts verborgen gehalten; als diese dann durch $\mathrm{Cn}$. Flavius bekannt gemacht seien, hätten die Rechtsgelehrten viele wortreiche, aber nichtssagende Formeln erfunden, welche nicht Jeder behalten konnte, um ihre Hände immer im Spiel zu behalten. Von Vorschlägen zur Verbesserung des Rechts ist nirgends in der Stelle die Rede. Die Worte: Fundus Sabinus meus est, immo meus, deinde judicium sollen meines Erachtens den nach Ciceros Meinung wesentlichen Kern der Vindikation im Gegensatz zu dem folgenden Wortschwall enthalten, sprechen also klar für die Nothwendigkeit der Eigenthumsbehauptung von beiden Seiten. In der nun folgenden Anführung der gebräuchlichen Formeln hätten danach die Worte: Fundus, qui est in agro, qui Sabinus vocatur, eum ego ex jure Quiritium meum esse ajo von beiden Parteien gesprochen werden miissen. Da es aber Cicero, nachdem er den Kern der Vindikation angedeutet hat, nicht darauf ankommt, eine vollständig getreue Darstellung des Ganges der Legis actio sacramento in rem zu 
seiner Zeit zu geben, wie die Stelle in ihrer Folge ergiebt, da er vielmehr nur die deductio in ihrer Anknüpfung an die vindicatio ausführlich darstellt, so lag es sehr nahe, dass er die zweimal vorkommende Formel der Vindikation nicht repetirte, er hätte sich ja sonst selbst der Weitschweifigkeit, welche er rügt, schuldig gemacht, sondern dass er die erste Vindikation einfach wegliess und lediglich die Kontravindikation, an welche sich dann die weiteren Formeln knüpften, citirte, zumal sein Haupttadel nicht gegen die Vindikation, sondern gegen die deductio gerichtet ist. Dass wir in den Worten Ciceros in der That die Kontravindikation vor uns haben, ergiebt sich daraus, dass die deductio, welche doch jedenfalls erst nach der Vindikation beider Parteien erfolgte, unmittelbar daran geknüpft ist, und dass durch den Satz: Quid huic tam loquaciter litigioso responderet ille, unde petebatur, non habebat ["Was er diesem so geschwätzig Klagenden (Wesentliches) erwiderte, hatte der Beklagte nicht"], die sämmtlichen vorher gesprochenen Worte als von ein und derselben Partei gesprochen dargestellt werden. Die Partei, welche die Kontravindikation spricht, wird hier ausdrücklich der Kläger genannt. Wenn nun überhaupt eine zweite Vindikation vorher gesprochen ist, so muss sie folgeweise der Beklagte gesprochen haben und ist er also auch nach dieser Stelle Vindikant, der Kläger Kontravindikant.

Auch die in jure cessio (Gai. II, 24) spricht für meine Meinung, denn der bereits im Besitz befindliche Erwerber spricht rem tenens die vindicatio. Also der Besitzer, welcher doch im Process Beklagter sein würde, vindicirt. Der Veräusserer, welcher den Besitz verloren hat, also klagen müsste, wird gefragt, ob er kontravindiciren will.

Durch die von mir entwickelte Erklärung der Eigenthumsbehauptung beider Theile erklärt sich auch die Stelle des Varro: L. L. V, 180: Qui petebat et qui infitiabatur . . . quingenos aeris ad pontem deponebant. Sie unterscheidet ganz klar zwischen Kläger und Beklagtem und indem sie den Ausdruck qui infitiabatur gebraucht, deutet sie gleichzeitig auf die günstigere Stellung des Beklagten im Beweisverfahren hin. Mit allen bisherigen Erklärungen der beiderseitigen Eigen- 
thumsbehauptung steht sie im Widerspruch, mit der von mir gegebenen ist sie aber völlig im Einklang.

Die Quellen sprechen nach Vorstehendem für die Richtigkeit der von mir aufgestellten Ansicht. Wäre sie richtig, so würden sich alle Schwierigkeiten von selbst heben. Es erklärt sich namentlich, weshalb der positiven Behauptung eine eben solche und nicht ein Leugnen entgegentritt, ohne dass deshalb die allgemeine Processregel, dass Beklagter sich auf die Vertheidigung beschränken kann, umgestossen werden muss. Die scheinbare Unvereinbarkeit dieser Processregel mit der Nothwendigkeit der beiderseitigen Eigenthumsbehauptung hat Lotmar in seinen die Schwächen der bisherigen Theorieen scharf kritisirenden Abhandlungen geleitet. Da aber die Nothwendigkeit dieser beiderseitigen Eigenthumsbehauptung aus den Quellen nicht wegzuinterpretiren ist, so musste nach einer anderen Lösung des Widerspruchs gesucht werden, wie er sie findet, und glaube ich, dass er sich löst ohne dass man durch Konjekturen etwas in die Quellen hineinzubringen braucht, was doch einmal nicht darin steht, sobald man davon abgeht, dass Vindikant, weil er zuerst spricht, Kläger sein muss, während es sich doch sehr wohl erklären lässt, dass bei der Vindikation ausnahmsweise Beklagter zuerst das Wort ergriffen hat. 